\title{
Nerve signaling explains spleen's fast, long-distance response to kidney infection
}

\section{Svava E. Steiner}

Ferdinand $X$. Choong

Haris Antypas

Carlos E. Morado-Urbina

Anette Schulz

Alex Bersellini Farinotti

Duygu B. Bas

Camilla I. Svensson

Agneta Richter-Dahlfors

Keira Melican

\section{Video Abstract}

Keywords: AIMES, a-hemolysin, IFNy, UPEC, pyelonephritis, inter-organ communication, nervous sensing, bacterial infection, antibiotics, antibiotic resistance, host-pathogen interaction, uropathogenic Escherichia coli, E. coli, kidney, spleen, inflammation, immune response, cytokine signaling, nervous system, neuro-immune communication

Posted Date: October 12th, 2021

DOl: https://doi.org/10.21203/rs.3.rs-966057/v1

License: (c) (i) This work is licensed under a Creative Commons Attribution 4.0 International License. Read Full License 


\section{Abstract}

Could doctors one day prescribe electrical stimulation to fight a bacterial infection? Work by an interdisciplinary team of researchers at AIMES suggests that might be possible. In line with the Goals of the United Nations' 2030 Agenda, researchers at AIMES are dedicated to promoting "Good Health and Well-Being" by achieving a better understanding of bacterial infections and the body's defenses against them. Some of their latest findings reveal a new aspect of host-pathogen interactions. In addition to the cascade of chemical signals that are activated when bacteria invade, the body might also conduct electrical signals across nerves-enabling the infected organ to call distant parts of the body to action. The team uncovered this form of "biological telecommunication" by studying rats with kidney infections caused by strains of E. coli. Within as little as 4 hours of infection, they could detect an immune response all the way in the spleen. Follow-up experiments confirmed that the detected response was not triggered by inflammation originating in the spleen. The short window of time during which these organs communicate raised the question: how does the spleen "know" so quickly that the kidney is infected? That inspired the team to look beyond chemical signaling and explore the possible role of nerve cells. Messaging through nerve cells is considerably faster than through chemical delivery. And growing evidence suggests that the nervous system plays an important role in the immune response. So-called "neuro-immune communication" explains how the immune system and the nervous system interact, and how bacteria and bacterial products can activate sensory neurons and modulate pain and local inflammation. But never before had researchers examined the functional outcomes of the neuro-immune communication pathway between the kidney and the spleen. The team from AIMES showed that a specific toxin expressed by E. coli called alpha-hemolysin could trigger nerve signals that traveled through the splenic nerve and into the spleen. The spleen responded by producing interferon gamma, a signaling molecule critical to the immune response. In models designed to mimic the tubular structure of the kidneys, interferon gamma could help modulate inflammation in infected kidney cells. As new knowledge often leads to new questions, more work is needed to understand how this form of neuro-immune communication occurs in humans during kidney infection. Working out those details could open up entirely new forms of medical treatment based on nerve stimulation instead of conventional drugs. With antibiotic resistance becoming a growing public health problem around the world, non-pharmaceutical treatment options would be a welcome addition in the fight against infection. 